\title{
Theoretische Grundlagen für Future Skills oder die "Drift to Self-Organisation“
}

Eine Vielzahl von theoretischen Bezügen aus verschiedenen Wissenschaftsdisziplinen können die zunehmende Bedeutung von Future Skills, als Handlungsfähigkeit in emergenten Kontexten erklären. Das Zusammenwirken komplexer Systeme führt zu Selbstorganisation und Systemveränderung. Wir bezeichnen diese Entwicklung als „Drift to Self-Organisation“. Diese Systemveränderungen sind davon gekennzeichnet, dass sie nicht linear auf den vorherigen Zustand zurückzuführen sind und nicht deterministisch zustande kommen, also keine Vorhersagen getroffen werden können. Vernetzung durch digitale Medien, globales Zusammenwirken und der Überschuss von Informationen durch die Digitalisierung führen zu schnelleren Veränderungen auf der Ebene der sozialen Organisationen die sich auf allen Ebenen der Makro-, Meso- und Mikroebene noch einmal selbst verstärken und beschleunigen. Der Zusammenhang der Ökosystemebenen führt dabei zur Beschleunigung selbstorganisierter Veränderung.

Future Skills ist ein schillernder Begriff, der derzeit Konjunktur hat, mehr durch seine programmatische Wirkung als durch seine konzeptuelle Kraft. Insofern ist er durchaus mit Begriffen wie Lebenslanges Lernen, E-Learning, Kompetenz oder Digitalisierung vergleichbar. Alles Begriffe, die oft für breite Entwicklungen standen und ganze Bündel theoretisch-konzeptioneller Bestandteile in sich verein(t)en.

Betrachtet man die derzeitige Forschung zum Thema Future Skills wird deutlich, dass dahinter ein ganz ähnlicher Diskurs wie beim Begriff des lebenslangen Lernens steckt. Programmatisch geht es darum, (Schlüssel)kompetenzen zu entwickeln, um die Innovationsfähigkeit von Arbeitsprozessen zu erhalten bzw. weiterzuentwickeln. Solche Begriffe treten wie Landmarken in der öffentlichen Debatte auf und zeichnen sich weniger durch klare begriffliche Schärfe als vielmehr durch ihre Orientierungswirkung aus. In diesem Kapitel stellen wir daher den aktuellen Forschungsstand zu wichtigen Theorien und Grundlagen für Future Skills dar. Dabei werden wir die Begriffe der Kompetenz, der Selbstorganisation und damit 
zusammenhängende Begriffe aufarbeiten. Hierbei wird auch der Begriff der Emergenz und der entstehenden Ordnung in selbstorganisierten Systemen thematisiert.

\section{B 2.1 "Drift to Self-Organisation": Selbstorganisation als gesellschaftliches Leitprinzip}

Selbstorganisation ist ein Prinzip, welches vielen gesellschaftlichen Entwicklungen zugrunde liegt und in vielen theoretischen Ansätzen als Erklärungsmodell herangezogen wird. Es entwickelt sich zu einem so durchdringenden Konzept, dass wir die Entwicklung zur Selbstorganisation als Leitprinzip mit dem Begriff „Drift to Self-Organisation" bezeichnet haben. Es stellt sich aber die Frage: Wie kann in Organisationen Kohärenz, Synergie und gemeinsames Handeln trotz oder gerade durch Selbstorganisation entstehen? Ist das nicht ein Widerspruch? Steht die Betonung des Selbst nicht einer kollektiven Ordnung entgegen?

\section{B 2.1.1 Selbstorganisation - Im Spannungsfeld von Struktur und Wandel}

Selbstorganisation ist eine disziplinenübergreifende Forschungsrichtung, die sich mit Systemen beschäftigt, die ohne externen Eingriff ordnungsbildend wirken.

\footnotetext{
"Intuitively, self-organization refers to the effect that a systems structure or organization appears without explicit control or constraints from outside the system. In other words, the organization is intrinsic to the self-organizing system and results from internal constraints and mechanisms, due to local interactions between its components." (Serugendo u. a. 2004:2)
}

Ordnungsbildung wird in so verschieden wissenschaftlichen Bereichen wie der Laserphysik, der Thermodynamik, der Evolutionsbiologie, der Meteorologie, der Informatik, den Wirtschaftswissenschaften und der Soziologie thematisiert. Dabei sind die Grundannahmen und Konzepte der Selbstorganisation so elementar unterschiedlich von denen der extern strukturierten, fremdbeeinflussten Ordnungssysteme, dass Paslack (2013) von einem Paradigmenwechsel spricht:

„Die Antworten, die auf diese und ähnliche Fragen gefunden wurden, gingen freilich über das spezielle Frageinteresse hinaus und begründeten eine völlig neue Sicht der Natur." (Paslack 2013) 
Die Forschungsrichtung der selbstorganisierenden Systeme begann sich in den sechziger Jahren zu etablieren. Dieser eigentlich recht späte Durchbruch des Selbstorganisationskonzeptes in der Wissenschaft liegt nicht zuletzt am Erfolg des mechanistischen Weltbildes in Kombination mit der mathematisch handhabbaren Theorie linearer Systeme, die eng mit der Differenzialrechnung verbunden ist. Dieser Erfolg führte dazu, Probleme so lange wie nur irgend möglich als linear zu klassifizieren, was den Blick auf nichtlineare Phänomene verwehrte. Durch die aufkommende Konzeption des Selbstmanagements in Organisationen, die digitale Vernetzung auf Mikro-, Meso- und Makroebene und eine Bewegung in Richtung hochemergenter systemischer Phasenverschiebungen sozialer Systeme, wurden lineare Modelle immer weniger erklärungsmächtig.

Selbstorganisation liegt als Prinzip vielen gesellschaftlichen Entwicklungen zugrunde. Es entwickelt sich zu einem so durchdringenden Konzept, dass wir die Entwicklung zur Selbstorganisation in der Gesellschaft insgesamt aber auch in den einzelnen gesellschaftlichen Teilbereichen, wie etwa den privaten oder öffentlichen Organisationen mit dem Begriff „Drift to Self-Organisation“ bezeichnet haben.

Der Clou an der Betrachtungsweise von Systemen als selbstorganisierten Entitäten ist das Phänomen der Dynamik. Dynamische Systeme sind notwendigerweise instabile Systeme. Die Strukturiertheit einerseits und Flexibilität dieser Systeme andererseits tritt aber nicht trotz, sondern gerade wegen ihrer Dynamik auf. Man kann als Schlussfolgerung formulieren: nur wandlungsfähige Systeme sind stabil und nur instabile Systeme sind wandlungsfähig. In Selbstorganisationsprozessen wirken Elemente in einem System auf bestimmte, aber unvorhersagbare Weise zusammen. Diesen Prozess, das Entstehen von neuen Eigenschaften oder Strukturen eines Systems infolge des Zusammenspiels seiner Elemente, bezeichnen wir als Emergenz (Stephan 2006; Stephan 2005).

Erpenbeck und Heyse (1999) weisen darauf hin, dass Unternehmensführung in der Praxis als ein Zusammenspiel von deterministischen Ansätzen und dem Gestalten von Rahmenbedingungen bezeichnet werden kann, die Mitarbeiterinnen und Mitarbeiter und Mitarbeitergruppen Freiheiten lassen, Entscheidungen und Handlungen im Rahmen des impliziten und expliziten Wissens zu treffen. Aufgabe eines wie auch immer gearteten Organisators in humanen, sozialen Systemen ist damit die Schaffung und Erneuerung von Kontexten, die die Freiheitsgrade oder Wahlmöglichkeiten erhöhen und damit das Potential für die Selbstregulierung und Innovation für alle Beteiligten vergrößern (Probst 1987:113). 


\section{B 2.1.2 Selbstorganisation als gesellschaftlicher Trend}

Das Prinzip Selbstorganisation ist die Grundorientierung der Next Organisations - der neuen Arbeits- und Lebensrealität der Menschen in immer größeren Teilen der Gesellschaft - der Next Societies. ${ }^{24}$ Als Prinzip liegt es vielen gesellschaftlichen Entwicklungen zugrunde. Es entwickelt sich zu einem so durchdringenden Konzept, dass wir die Entwicklung mit dem Ausdruck „Drift to Self-Organisation“ bezeichnen.

Abseits institutioneller Akteure und politischer Vorgaben scheint es, gekoppelt mit einem höheren Grad an Selbstverantwortung zunehmend Raum zu greifen. Als Ergebnis entstehen heute erste Formen von Aktivitäten eines "progressiven Wirs“ wie Kruse (2009) sie bezeichnet und sie als die Fortsetzungen der neuen sozialen Bewegungen der Neunziger beschreibt. Es entstehen dabei andere und neue Formen von Gemeinschaft und Solidarität. Selbstorganisation als zentraler Begriff der Future Skills und der zukünftigen Arbeitswelt kann je nach Perspektive auch schnell als neoliberales Agieren verstanden werden - gerade wenn er ohne schützendes Netz versehen ist. So beschreibt etwa der Ökonom und Soziologe Oliver Nachtwey den Übergang zum neu gestalteten deutschen Sozialstaat in der „regressiven Moderne“ jenseits des „paternalistischen Führsorgeprinzip" und benennt Selbstorganisation und Eigenverantwortung als die zunehmend dominanten Begriffe (Nachtwey 2016). Es ist wichtig, dass jede Zukunftsvision, die Selbstorganisation und Selbstverantwortung in den Mittelpunkt stellt, auch gleichzeitig diese politisch gesellschaftlichen und sozialen Zusammenhänge nicht aus dem Blick verliert.

Klaus Schwab, der Chef des Weltwirtschaftsforums, untersucht in seinem Buch „Die Vierte industrielle Revolution“ deren potentielle Auswirkung auf Unternehmen, Staaten, Länder, die Gesellschaft und den Einzelnen. Er betont, dass Selbstorganisation die gravierendste Auswirkung der Digitalisierung darstellt: „Eine der weitreichendsten Veränderungen in all diesen Bereichen wird auf eine einzelne Kraft zurückzuführen sein: Empowerment.“ (Schwab 2016) Die Ermächtigung bzw. Befähigung zur Selbstbestimmung verändere alles: das Verhältnis zwischen dem Staat und seinen Bürgern, zwischen Unternehmen und ihren Mitarbeiterinnen und Mitarbeitern, zwischen Anteilshaltenden sowie Kundinnen und Kunden, zwischen Supermächten und kleineren Ländern. Damit tritt zu den rein systemischen Betrachtungen der Ökosystemtheorie Bronfenbrenners (1981), die zwischen den unterschiedlichen Ebenen (der Mikroebene, der Mesoebene und der Makroebene) sich gegenseitig beeinflussende systemische Zusammenhänge betont, noch eine neue Qualität hinzu. Die Qualität nämlich, dass die in den unterschiedlichen Ebenen handelnden Akteurinnen und Akteure durch eine neue Orientierung der

24 Zum Begriff siehe auch https://next-society.de 
Selbstorganisation und Eigenverantwortung eine neue Unvorhersehbarkeit und Unsicherheit produzieren. Die disruptive Wirkung dessen, was Schwab „Die Vierte Industrielle Revolution“ (2016) nennt, wird es nötig machen, dass sich Akteurinnen und Akteure als Teile eines weitverzweigten Systems verstehen, das nur mit kooperativen Formen der Interaktion erfolgreich sein kann. In ihrer Studie „Next Germany“ fassen Bühl et al. es so:

\begin{abstract}
„Diese Systeme sind ihrer Natur nach nicht mehr auf lokale oder regionale Zusammenhänge begrenzt, sondern sind auf unterschiedlichsten Ebenen kommunikativ und prozessual miteinander verwobene Organisationen oder soziale Systeme, die sich gegenseitig in ihren digitalen Abläufen beschleunigend beeinflussen." (Brühl, Koppel, Schomburg \& Schuldt 2017)
\end{abstract}

Selbstorganisation als Prinzip, Selbstverantwortung als Impuls von Innen und aktive Einmischung als Ausdruck einer wachsenden Ungeduld - so beschreibt Handelsblatt-Herausgeber Gabor Steingart in seinem Buch „Weltbeben. Leben im Zeitalter der Überforderung" (Steingart 2016) den Zeitgeist der Next Society. Im Kapitel zur Demokratie, mit dem Untertitel „Aufstand der Bürger“ heißt es:

\begin{abstract}
„[D]er kommende Aufstand wird einer sein, der den Westen mehr verändert als alle Wahlen der vergangenen Jahrzehnte. Im Zentrum dieser Veränderung stehen keine Partei oder Religion, kein Führer oder Guru, sondern ein selbstbewusstes Bürgertum, das den Umsturz je nach Verhältnissen will, die als widrig empfunden werden." (Steingart 2016)
\end{abstract}

Die derzeit im Jahr 2019 allfreitäglich stattfindenden Demonstrationen der Schülerinnen und Schüler gegen die Klimakatastrophe lassen diese Diagnose in neuem Lichte als richtig erscheinen. ${ }^{25}$ Steingart berichtet, es gehe darum, die Verfahren der Gewinnung und Ausübung von Macht grundsätzlich zu verändern: Transparenz, Teilhabe, Kommunikation und Mitbestimmung sieht er als die Leitbegriffe dieser stillen Revolution. Diesmal werde sich die Verdrossenheit nicht im Leerlauf der eigenen Befindlichkeit drehen, sondern als Veränderungsenergie wirksam werden. Insgesamt zeigt sich in nun historisch einmalig deutlicher Weise ein neues Bewusstsein: Selbstorganisation und Eigenverantwortung sind das neue Grundprinzip für die Funktionsweise von gesellschaftlichen Systemen und Organisationen. Sind es bisher oftmals noch als gegensätzlich empfundene oder thematisierte Pole der gesellschaftlichen und organisationalen Entwicklung, entweder die expansive neue Entwicklung („alles wird selbstständig Bottom-up gestaltet“) oder die restriktive

25 https://fridaysforfuture.de 
Gegenbewegung („es ist wichtig, dass jemand Top-down, von Oben die Dinge in die Hand nimmt“) gewesen, so lösen sich diese scheinbar gegensätzlichen Pole immer weiter in Richtung synergetischer Gestaltung auf.

Heutige Organisationen finden sich in diesem Spannungsfeld wieder. Einerseits sind stabile Strukturen, Abteilungen und zielüberdauernde Zielsetzungen wichtig für kontinuierliche Entwicklung, andererseits besteht die Notwendigkeit, immer mehr Steuerung durch agile, sich schnell ändernde dezentrale Organisationseinheiten zuzulassen und zu befördern. Beide Pole gleichzeitig in den Blick zu nehmen und die dadurch entstehende Spannung aufrechtzuerhalten, ohne sie scheinbar einfach aufzulösen, ist der neue Balanceakt, den Organisationen und Gesellschaften zu leisten haben. Oft stehen hierbei, je nach Blickwinkel, entweder Ohnmacht oder Hierarchiegläubigkeit im Vordergrund - oder der Aufbruch in ein neues spannendes Miteinander.

\section{B 2.1.3 Strategien und Organisationsformen für Selbstorganisation}

Selbstorganisationsstrategien sind dabei ein adäquates, geeignetes Mittel für unsere Zeit. In seinem Buch „Beschleunigung“ benutzt der Soziologe Hartmut Rosa den Begriff „Driften“ als mögliche „Reaktion spätmoderner Subjekte auf die komplexe tosende Welt“ (Rosa 2005: 379ff). Der Drifter lasse sich einfach vom Strom des Lebens mitreißen, wolle nicht kontrollieren, planen und steuern, sondern stattdessen ein situatives Selbst entwickeln. Das stellt die berechtigte Frage in den Raum, welche Wirkung und welchen Verbreitungsgrad selbstorganisierte Individuen mit einem großen Grad an Selbstverantwortung haben können. Wie steht es um Dauer und Verbindlichkeit solcher Ansätze für eine langfristige Entwicklung und was bedeuten sie eigentlich in der Praxis?

Betrachtet man den derzeitigen Stand moderner Managementliteratur, so wird schnell deutlich, dass neue Formen der Organisation und des Managements unter dem Prinzip Selbstorganisation ausprobiert werden und wir uns weltweit in einem Experimentierfeld befinden. Die meisten Beobachterinnen und Beobachter, die über neue Formen der Organisation, des Selbstmanagements, der Selbstorganisation und der Eigenverantwortung schreiben, unter Stichworten wie Holokratie ${ }^{26}$, demo-

26 Holokratie - auch Holokratie - ist ein Kompositum aus holos (altgriechisch für vollständig, ganz) und kratía (altgriechisch für Herrschaft) und ist ein System zur Entscheidungsfindung welches dem Unternehmer Brian Robertson aus Philadelphia (USA) in seiner Firma Ternary Software Corporation zugeschrieben wird. Es geht dabei darum, 
kratische Organisation, soziokratisches Management oder auch über andere Typen selbstorganisierter Organisationen, richten darüber sehr pointiert und nehmen extreme Sichtweisen ein. Entweder werden die chef- und führungslosen flachen Hierarchien und Arbeitsumgebungen für ihre Flexibilität und das Engagement gelobt oder sie werden als naive soziale Experimente verdammt, die ignorieren wie Dinge wirklich gemacht werden müssen.

Die Wahrheit liegt wie so oft dazwischen, im Zentrum des Spannungsfeldmanagements. Um mehr akkurate, balancierte Perspektiven einzunehmen, ist es wichtig, einmal hinter die Buzzwords zu schauen, die diese neuen Strukturen beschreiben - postbürokratisch, poststrukturalistisch, digital, organisch, usw. Es geht darum, zu untersuchen, welche neuen Formen sich gebildet haben und auf welcher Basis sie funktionieren. Sowohl in den Mühen der Niederungen und in den Schützengräben der operativen Organisationen als auch auf der Ebene der organisationsweiten Strategiebildung und Policy-Entwicklung.

In der allgemeinen Debatte um neue Organisationsformen werden immer wieder extreme Positionen eingenommen, Evangelisten schlagen sich auf die eine oder andere Seite. Dabei geht es darum, zunächst einmal wertfrei zu betrachten, welche Grundpositionen es gibt, was für Strukturierungskonzeptionen daraus erwachsen und wie diese wirken bzw. wie angemessen sie für die unterschiedlichen Organisationsanforderungen sind. Die Diskussion zentriert sich um zwei Gegensatzpaare, die die Pole des Spannungsfeldes bilden welchem heutige Organisationen ausgesetzt sind. Verlässlichkeit (reliability) auf der einen Seite und Anpassungsfähigkeit (adaptability) auf der anderen Seite. Dabei bedeutet Verlässlichkeit als Prinzip eine Vielzahl an Dingen wie beispielsweise vorhersagbare Gewinne für Shareholder zu generieren, sich an Regeln zu halten, compliant zu sein, stabile Arbeitnehmeranforderungen und Mitarbeiterzahlen zu haben und nicht zuletzt die Kundenanforderungen und die Anforderungen der Klienten und Stakeholder im öffentlichen Bereich zu erfüllen. Anpassungsfähigkeit auf der anderen Seite bedeutet in Situationen situativ handeln zu können, abseits der Strukturprinzipien und Regeln, flexibel in der Lage zu sein, kleine Anpassungen in der Produktion oder dem Herstellungsprozess und den Services zu machen, um lokale Anforderungen zu erfüllen, aber auch größere strategische Umsteuerungen und strukturelle Anpassungen vornehmen zu können. Organisationen stehen immer im Spannungsfeld zwischen Stabilität und Anpassungsfähigkeit, aber meistens werden sie als Gegensatzpaare im entweder-oder angesehen und nicht als Pole, die ein organisationskulturelles Spannungsfeld aufzeigen, ein spannungsgeladenes Miteinander. In den Interviews der Future Skills

alle Organisationsebenen mit größtmöglicher Transparenz und partizipativen Beteiligungsmöglichkeiten auszustatten. 
Studie zeigt sich jedoch, dass in der Wahrnehmung der Beteiligten oftmals eins das andere ausschließt. Es besteht Unsicherheit darin, ob nicht zu viel Betonung der Anpassungsfähigkeit Fragmentierung erzeuge und zum Verlust der Vorteile führe, die mit Fokussierung und Skalierung einhergehen. Und obwohl managerielle Hierarchien sich oft in allen Richtungen irren können, so sind sie doch oft starke Befürworter der eher stabilen, hierarchischen Organisationform. Dabei sind Mitarbeiterinnen und Mitarbeiter genauso auf Stabilität und Verlässlichkeit angewiesen, wie auf Flexibilität und Anpassungsfähigkeit. Um ihre Arbeit effektiv zu verrichten, müssen sie in einer stabilen Arbeitsumgebung arbeiten, Zugang zu den kritischen Arbeitsmitteln und klare Zielvorstellungen und Verantwortlichkeiten haben. Aber genauso benötigen sie auch einen Raum, in dem Anpassung an sich verändernde Bedingungen möglich ist und ad hoc Entscheidungen getroffen werden können. Denn managerielle Hierarchien versagen oft darin, die notwendige Flexibilität bereitzustellen. Unter dem Stichwort „adhocracy“ thematisiert Friedrich Lindenberg seit 2016 diesen Umstand in der jüngsten Entwicklung. ${ }^{27}$ Als Manager ist es nicht einfach, die richtige Balance zwischen Verlässlichkeit und Anpassungsfähigkeit zu finden. Daher entwickeln sich in jüngster Zeit Ansätze des Selbstmanagements, der dezentralen Organisation, der vernetzten Organisation mit flachen Hierarchien oder weitergehende Ansätze unter dem Stichwort Holokratie, Soziokratie, demokratische Organisation oder Adhocracy als neues großes Experimentierfeld dynamischer Organisationen in sich schnell verändernden Umfeldern.

\section{B 2.1.4 Self-Management als Vorläufer von Selbstorganisation}

Dabei gibt es Selbstorganisation als Organisationsform schon eine ganze Weile. Erst durch die Industrialisierung ist der ursprünglich ganzheitliche Arbeitsprozess in Teilschritte unterteilt und im bekannten Industrialisierungsprozess dann in kleinste Produktionsfertigungs- und Wertschöpfungseinheiten untergliedert. Tatsächlich beginnt aber die Zeit der Selbstorganisation schon vor langer Zeit; nämlich als vor ca. 65 Jahren Eric Trist ${ }^{28}$ - ein Mitglied des britischen Tavistock Institute - beobachtete,

27 Friedrich Lindenberg hat im Rahmen seiner Bachelorarbeit eine Open-Source-Software namens Liquid Democracy zur Onlinebeteiligung für Organisationen und Institutionen entwickelt.

28 Eric Lansdown Trist war ein führender britischer Sozialpsychologe auf dem Gebiet der Organisationsentwicklung. Er war Mitgründer des Tavistock Institute of Human Relations in London. 1949 publizierte Trist einen bekannten Artikel „Some Social and Psychological Consequences of the Longwall Method of Coal Getting (Trist, Bamforth 1951) über seine Arbeit zur Organisationstheorie in einer englischen Kohlenmine 
dass Teams die nach Selbstmanagementprinzipien arbeiten, substanziell ihre Produktivität im Kohleabbau erhöhen konnten (Trist \& Bamforth 1951). Damals war es die nichthinterfragte Standardvorgehensweise, den Kohlebergabbau als kleinschrittigen Prozess durchzuführen. Jedes Team arbeitete nur an einem kleinen Schritt und die Schritte wurden nacheinander durchgeführt. Das Modell basierte auf Frederic Tailors Managementansatz und Henry Fords Fließbandkonzeption. Ein Team musste die Schicht beenden, bevor das nächste anfangen konnte. Aber die Bergarbeiter in South Yorkshire in England begannen ihre Arbeit spontan und selbstorganisiert anders zu organisieren. Es bildeten sich autonome Arbeitsgruppen, die mit umfassenden Fähigkeiten ausgestattet waren, die wechselnde Rollen übernahmen und Schichten mit einer nur minimalen Anleitung und Supervision, die 24 Stunden am Tag Kohle fördern konnten ohne auf die Arbeitsergebnisse der davorliegenden Schicht zu warten. Sogenannte „Self-Managed Teams“ (SMT) gewannen in der Folge an Popularität. In den siebziger und achtziger Jahren des letzten Jahrhunderts wurden immer mehr Versuche unternommen, diese Form des Managements einzuführen. In Europa entstand das partizipative Management (Sexton 1994). Auch das Konzept der sogenannten „industrial democracy“ wurde eingeführt (Korsch 1968). ${ }^{29}$ In Japan entwickelten sich diese Konzepte in Qualitätszirkel und kontinuierliche Verbesserungskonzeptionen (KVP). In den USA entstanden aus diesen Konzepten die Organisationsprinzipien für sogenannte Innovation Task Forces.

Die Entwicklung Richtung „Self-Managed Teams“ verhalf vielen Organisationen und Unternehmen zu Durchbrüchen in der Herstellungs- und Serviceausübung. So

in Yorkshire. Aus diesen Untersuchungen entstanden der Tavistock-Ansatz und die soziotechnischen Forschungsmethoden. Im soziotechnischen System wurden das technische und das psychosoziale System miteinander verknüpft. Zusammen mit Fred Emery entwickelte Trist den soziotechnischen Zugang zum „Work Design“ - einer Anwendung der Organisationsentwicklung zur sogenannten Humanisierung der Arbeit (Verbesserung der Arbeitszufriedenheit, -effizienz, -qualität, Absentismus usw.): Intern geführte, selbstregulierende Arbeitsgruppen, würden produktiver und motivierender für die Arbeiter sein, als die bisherige konventionelle Hierarchie.

29 Der deutsche Marxist Karl Korsch hat nach einem längeren Aufenthalt bei den Fabiern in London (1912/13) den auf Sidney und Beatrice Webb zurückgehenden Begriff Industrial Democracy erstmals ins Deutsche übersetzt (Die Fabianische Gesellschaft, gegründet im Januar 1884, ist eine britische, sozialistische intellektuelle Bewegung, die durch ihre wegweisende Arbeit im späten 19. Jahrhundert bis zum Ersten Weltkrieg bekannt wurde. In seiner Schrift „Arbeitsrecht für Betriebsräte“ (1922) verwandte er nicht nur den Terminus „Industrielle Demokratie“, sondern erweiterte auch seinen Inhalt. Während die Fabier vorwiegend an Selbstverwaltung/ Mitbestimmung/ Partizipation der Arbeitenden im Betrieb und Unternehmen dachten, schloss Korsch auch die überbetriebliche Ebene z. B. in Form von Wirtschafts- und Sozialräten auf sektoraler und gesamtwirtschaftlicher Ebene mit ein. 
konnte die Volvo Fabrik in Kalmar in Schweden ihre Produktionsdefekte 1987 um 90 Prozent reduzieren. FedEx konnte Servicefehler 1989 um 13 Prozent reduzieren. In den späten achtziger und frühen neunziger Jahren entwickelten C\&S Wholesale Grocers ein neuartiges Lagerhauskonzept mit Self-Managed Teams welches 60 Prozent Kostenvorteile den Wertbewerbern gegenüber bot. General Mills konnten ihre Produktivität um 40 Prozent in den Fabriken steigern, die Self-Managed Teams einführten. Diese Teams wurden in den 1990zigern immer bekannter. Von dem Konzept wurden sich Vorteile im Bereich höherer Produktivität versprochen, insbesondere in solchen Arbeitsfeldern, die komplex und dynamisch waren.

In den Organisationen, in denen sie eingeführt wurden, war jeweils nur ein Bruchteil der Angestellten in die Konzeptionen des Self-Managements involviert. Meistens in Bereichen in denen Anpassungsfähigkeit wichtiger war als Stabilität und Verlässlichkeit. Mit der Zeit entwickelten sich diese Arbeitsumgebungen weiter zu Arbeitsökosystemen in denen Mitarbeiterinnen und Mitarbeiter auf einfache Weise ihre eigene Leistung überprüfen konnten und iterativ verbesserten. Mit der Zeit kam die Frage auf, warum Self-Management nur auf Teamebene eingeführt werden soll? Immerhin schien es so, als würden die stark überformenden Organisationsstrukturen, teilweise als Matrix aufgebaut, teilweise sehr hierarchisch und komplex mit übergreifenden Reporting Schemata, die Entwicklung solcher Self-Managed Organisations Units behindern. Der C\&S Geschäftsführer Rick Cohen berichtet, dass bei der Arbeit mit Self-Managed Teams das Schwierigste sei, die Manager außen vorzuhalten und die Teams das tun zu lassen, was notwendig sei (DeLong et al. 2003). Die Frage stellte sich also, warum nicht gesamte Organisationen auf den Prinzipien des Self-Managements aufgebaut würden.

\section{B 2.1.5 Selbstorganisation als Managementphilosophie}

Und tatsächlich haben Organisationen angefangen in diese Richtung zu gehen. Management Vordenker wie Warren Bennis und Henry Mintzberg, der in seinem berühmten Artikel im Harvard Business Review schon 1981 die Frage stellt „Organization Design: Fashion or Fit?", haben bereits in den achtziger Jahren einen Wandel in Richtung neuer Strukturen, die als Adhocracy bezeichnet werden, bemerkt: flexible informelle Managementstrukturen. Eine Dekade später wurde das Internet selbst zum Modell für die Konzeption der sogenannten „networked firm“, der virtuellen Unternehmen. Mit der Free-Software-/ „Open Source“-Bewegung“ im Jahr 1983, 
dem Aufkommen von agilen Arbeits- und Planungsmethoden, wie „Scrum ${ }^{“ 30} \mathrm{im}$ Jahre $1986^{31}$ sowie Sharing Economy Plattformen und Geschäftsmodellen wie dem von Gerrett Camp und Travis Kalanick ursprünglich als Limousinenservice gegründeten Uber im Jahr 2009 oder der 2008 von Brian Chesky, Joe Gebbia und Nathan Blecharczyk in San Francisco gegründeten Unterkunftsplattform Airbnb, setzen sich partizipative und responsive Organisationsstrukturen in vielen Bereichen immer weiter durch. Holokratie, Podularity (ein Konzept von Dave Gray aus dem Jahr 2013 mit Wurzeln in der agilen Softwareentwicklung, publiziert in Gray, Vander Wal (2014)) und viele darauf aufbauende organisationsspezifische Variationen von Selbstorganisation kommen hinzu. Diese neuen Formen stellen sich gegen hierarchische Managementkonstrukte und -prinzipien. Aber in bestimmter Weise und im Gegensatz zur öffentlichen Wahrnehmung, ähneln sie dem Konstrukt der Bürokratie wie Max Weber es im frühen 20. Jahrhundert definiert hat (Weber 1921). Bürokratie, so definierte er, verortet Autorität in depersonalisierten Regeln und Rollen und nicht in Status, Klasse oder Reichtum. Die Idee von Weber war, Bürokratie als Konzept aufzufassen, in dem Individuen von dem diktatorischen Recht schlechter Chefs befreit werden. Self-Management Systeme zielen auf dieselbe Sache, nur mit weniger Starrheit. In gewisser Weise könnte man sie als Bürokratie 2.0 verstehen.

\section{B 2.2 Kompetenz als Grundlage für selbstorganisiertes Handeln}

In diesem Kapitel wird die Bedeutung von Kompetenz als Grundlage für selbstorganisiertes Handeln beschrieben. Vom Lernen zur Bildung, vom Wissen zur Kompetenz. Der Kompetenzbegriff ist seit langer Zeit in der Erziehungswissenschaft und Psychologie verankert. In der Erziehungswissenschaft wurde er von Heinrich Roth (1971) eingeführt, in der Psychologie geht er auf Franz Weinert (2001) zurück. Seine unterschiedlichen Definitionen eint ein gemeinsamer Kern: Zunächst sehen alle Definitionen vor, dass es unterschiedliche Teilbereiche von Kompetenzen gibt - die

30 Scrum (englisch für „Gedränge“) ist ein Vorgehensmodell des Projekt- und Produktmanagements, insbesondere zur agilen Softwareentwicklung. Es wurde ursprünglich in der Softwaretechnik entwickelt, ist aber davon unabhängig. Scrum wird inzwischen in vielen anderen Bereichen eingesetzt. Es ist eine Umsetzung von Lean Development für das Projektmanagement.

31 Scrum wurde als Begriff erstmals 1986 im Harvard Business Review erwähnt, in einem Artikel von Nonaka und Takeuchi (1986) zum Thema The New Product Development Game. 
wir hier als Kompetenzfelder bezeichnen - beispielsweise Sozialkompetenz, Personalkompetenz, Fach- und Methodenkompetenz. Diese wiederum, enthalten ihrerseits weitere Kompetenzen. Darüber hinaus umfassen alle Kompetenzbegriffe zweitens eine Anzahl von Faktoren, die sie mit Handlungen verknüpfen, wie beispielsweise kognitive Faktoren - also das handlungsrelevante Wissen, Volition - als den Willen zu einer Handlung, Motivation - als extrinsische und intrinsische Beweggründe für eine Handlung, soziale Faktoren einer Handlung und wertbezogene Faktoren in einer Handlungssituation. Drittens gehen alle Kompetenzkonzepte davon aus, dass Kompetenzen durch Lernen erwerbbar sind. Und viertens, dass sie über das reine Reproduzieren von Abläufen hinaus, die Fähigkeit einer Person beschreiben, unbekannte Probleme in unvorhersehbaren, komplexen Problemsituationen zu lösen. Mit Erpenbeck definieren wir Kompetenz hier folgendermaßen:

„Kompetenzen sind die Fähigkeit in offenen Problem- und Entscheidungssituationen selbstorganisiert und kreativ zu handeln. Kompetenzen sind Selbstorganisationsdispositionen." (Erpenbeck in Faix et al. 2012)

Die Definition macht noch deutlicher, welche bedeutende Rolle Kompetenz für Future Skills spielt, als die zuvor beschriebenen Eigenschaften dies schon nahelegen. Denn sie ist auf zukünftige unbekannte Handlungen gerichtet und spricht zudem von einer Handlungsdisposition - also nicht einer feststehenden vordefinierten Fähigkeit. Diese Disposition zu einer selbstorganisierten Handlung macht das Konzept aus bildungswissenschaftlicher und lernpsychologischer Sicht so fruchtbar für das Konzept der Future Skills. Future Skills stellen daher spezifische Kompetenzen im beschriebenen Sinne dar. Im Konzept der Future Skills wird der Begriff der Kompetenz gewissermaßen angewandt und auf den Bereich von emergenten Handlungskontexten bezogen. Dieses sind genau solche Handlungskontexte, in denen unvorhergesehene, neu entstehende Zusammenhänge zu verstehen und unvorhersehbare Problemlagen zu bewältigen sind. Im Begriff der Unvorhersehbarkeit ist auch bereits Selbstorganisation angelegt. In der NextSkills Studie konnten Hinweise gesammelt werden, die zeigen, dass in Organisationen zukünftig eine Dominanz von Selbstorganisationsdispositionen gegenüber vorbereiteten Lösungsansätzen gefordert werden wird.

Das Verständnis einer selbstorganisierten Handlungsfähigkeit, wie es im Future Skills Ansatz angelegt ist, zielt auf die Fähigkeiten von Menschen ab, in zukünftigen, unsicheren, vorher unbekannten Kontexten erfolgreich handeln zu können. 


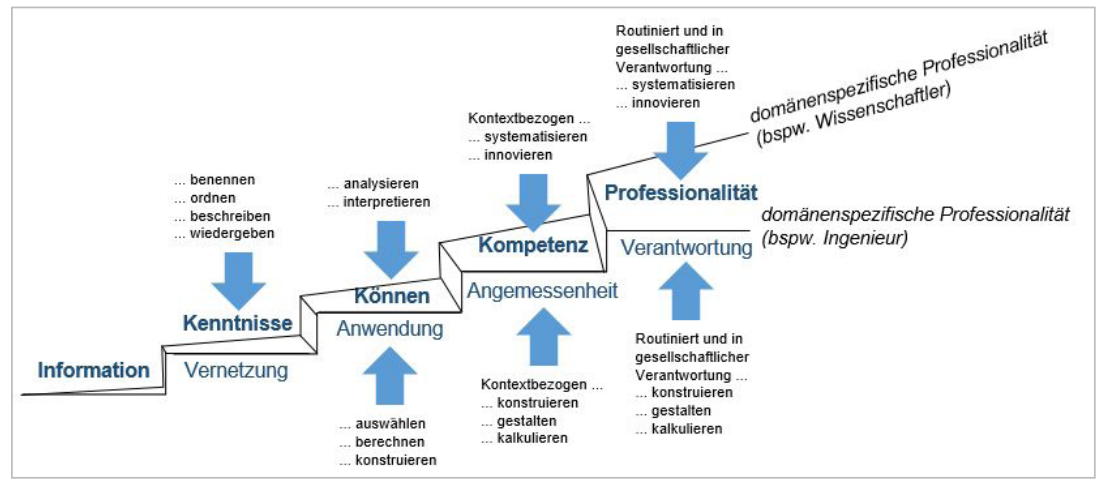

Abb. 21 Stufenmodell professioneller Kompetenz (Quelle: Wildt 2006)

Dabei ist es wichtig, zu verstehen, dass sich Wissen und Kompetenz, Handlung und Professionalität nicht gegenseitig ausschließen oder alternative Konzepte darstellen. Vielmehr sind sie im Konzept von Kompetenz integriert. So zeigt Abbildung 21, dass Wissen lediglich auf einer niedrigeren Stufe angesiedelt ist als Kompetenz und Professionalität, jedoch einen notwendigen Schritt auf dem Weg zu Kompetenz und Professionalität darstellt.

In Anlehnung an Erpenbeck (2007) schlagen wir abschließend vor, Selbstorganisation als eine eigenständige Kompetenz anzusehen, die die Qualität hat, andere Kompetenzen miteinander zu verbinden, also zur Interaktion und Kombination von Kompetenzen zu dienen. Diese Vorstellung bedeutet, die unterschiedlichen Kompetenzfelder in einer bestimmten Relation zueinander zu sehen. Kompetenzen zur Handhabung der organisatorischen Voraussetzungen, Kompetenzen zur Zusammenarbeit und Kommunikation, als auch Kompetenzen zur Prioritätensetzung und Koordination haben „Mittelcharakter“ (auch Erpenbeck \& Heyse 1999). Sie dienen zur Zielerreichung von Wertschöpfungshandlungen, zur Handhabung von Störungen, zur Qualitätsarbeit und der Handhabung der physischen Umgebungsbedingungen - das heißt also auch dazu, andere Kompetenzen besser nutzen zu können. Aufbauend auf diesen Überlegungen und in Anlehnung an Untersuchungen von Erpenbeck und Heyse (1999) folgern wir, dass Selbstorganisationskompetenz und deren Entwicklung sich folgendermaßen manifestiert:

- Selbstorganisation als eine besondere Qualität von Kompetenz, die neben anderen Kompetenzen (z. B. Fach-, Methoden-, Sozial- oder Selbstkompetenz) existiert und/oder 
- Selbstorganisation als ein Niveau (Stufe) von Kompetenz; d.h. als ein Grad von Expertise, der in allen Kompetenzfeldern (z. B. Fach-, Methoden- usw.) vorkommen kann und/oder

- Selbstorganisation als eine Kompetenz, die die Entwicklung von einem Kompetenzniveau zum nächsten beschreibt und/ oder

- Selbstorganisation als eine besondere Form der Interaktion und Kombination (des Interaktions- und Kombinationshandelns) zwischen verschiedenen Kompetenzbereichen.

\section{Exkurs: Mythen und Missverständnisse zu Kompetenz und Kompetenzlernen in der Hochschule}

Im Bereich der Kompetenzdiskussion gibt es viele Mythen, Miss- und Unverständnisse. Kompetenz könne doch nicht das Ziel von Lernen in der Schule oder im Studium sein, vielmehr müsse sich auf Wissen fokussiert werden, sonst würden die Lernenden doch nichts Substantielles lernen. Kompetenz sei, wenn überhaupt, etwas für die höheren Semester in einem Hochschulstudium, wenn das Wissen bereits vermittelt sei und man dann auch Kompetenzen erlernen könne. Ein zweiter Mythos liegt darin, dass Kompetenz außerhalb der eigentlichen Themengebiete erworben werden könne - beispielsweise in einem Seminar für Schlüsselkompetenzen. So haben viele Hochschulen es bis heute verstanden, ihre Studiengänge kompetenzorientierter zu gestalten, indem sie zusätzlich Lehrveranstaltungen für Kompetenzen oder Schlüsselkompetenzen eingeführt haben, während sich aber in den eigentlichen Lehrveranstaltungen nichts änderte. Ein drittes Missverständnis besteht darin, dass der Kompetenzbereich sowieso sehr unklar bis esoterisch sei und es nicht klar abgegrenzt werden könne, was eigentlich unter Kompetenz verstanden, geschweige denn operationalisiert werden könne, wie diese im Lehrgeschehen zu vermitteln sei.

In vielen Diskussionen wird jedoch auch deutlich, dass die Kompetenzdiskussion als Zielvorstellung bereits tief in das Bewusstsein der für Bildung Verantwortlichen eingedrungen ist und sich derzeit in einer Gestaltungsphase befindet, in der sortiert, verworfen und neu geplant wird. Kompetenzorientierung erfordert nämlich tatsächlich ein völliges Umdenken der bisher stark vermittlungsorientiert aufgebauten Lehrphilosophie. 


\section{B 2.3 Bildungstheoretische Fundierung des Future Skills Konzepts}

Bildung ist der Prozess, der zur Entwicklung von Future Skills führen soll. Doch wie ist das zu verstehen? In welcher besonderen Weise kann das Konzept der Future Skills auch bildungstheoretisch verstanden werden?

Zunächst einmal ist zu erkennen, dass die am pädagogischen Prozess beteiligten Akteurinnen und Akteure - Lernende, Professionelle, Management/ Organisation und Staat/ Gesellschaft - unterschiedliche Perspektiven und Kriterien hinsichtlich der Qualität von Bildungsergebnissen haben, also dem, wie Future Skills ausgestaltet sein sollen. Die unterschiedlichen Perspektiven lassen es unwahrscheinlich erscheinen, dass Bildung automatisch zur Ausbildung von Future Skills führt. Grundsätzlich argumentieren wir im Rahmen eines Mittel-Zweck-Verhältnisses, also entsprechend der Vorstellung, dass Bildungsmittel so gestaltbar sind, dass es dem Zweck der Entwicklung von Future Skills entsprechen könnte. Die Entwicklung von Future Skills ist damit eine Frage nach den Mitteln und Methoden, mit deren Hilfe Bildungsvorgänge bei den Nutzerinnen und Nutzern von Bildungsdienstleistungen (im Folgenden als Lernende bezeichnet) durch spezifische (pädagogische) Vermittlungsformen im weitesten Sinn angeregt und Future Skills erworben werden.

Neben der Frage der Zweck-Mittel-Relation steht eine weitere, nämlich danach, ob es auch normativ sinnvoll ist, Future Skills zu vermitteln. In Bezug auf diese Frage wird davon ausgegangen, dass durch die sozial- und bildungspolitisch ausgestalteten Leistungen in einem demokratisch legitimierten Staat eine materielle, soziale und geistige Teilhabe an den gesellschaftlichen Entwicklungen garantiert werden soll. Eine solche Teilhabe ermöglicht Bürgerinnen und Bürgern ein weitgehend selbstbestimmtes und selbstverantwortliches Leben. Teilhabe und Teilnahme (Partizipation) an gesellschaftlichen Gestaltungsprozessen ist hierfür ein konstituierendes Merkmal. Partizipation kann in dieser Hinsicht als die Sicherstellung von gesellschaftlichen Gestaltungsmöglichkeiten verstanden werden. Ist Partizipation am gesellschaftlich-demokratischen Prozess durch extern bedingte, materielle, finanzielle oder soziale Problemlagen gefährdet, so findet eine politisch initiierte Unterstützung statt. Im Bildungsbereich kommen dort pädagogische Dienstleistungen zum Einsatz, wo die subjektiven Fähigkeiten und Kompetenzen der Bürgerinnen und Bürger entweder (neu) aufgebaut, in einer defizitären oder gefährdeten Form vorliegen oder teilweise verlorengegangen sind.

Pädagogische Handlungszusammenhänge, die Bildungsvorgänge auslösen sollen, enthalten immer Momente der (1) Ermöglichung, (2) der Erhaltung und (3) der Wiederherstellung von Fähigkeiten und Kompetenzen, die Partizipationsmöglichkeiten eröffnen. Innerhalb der verschiedenen Bereiche der Pädagogik sind diese Momente 
allerdings unterschiedlich gewichtet: so zielen Maßnahmen, die die Einführung der nachwachsenden Generation in das gesellschaftliche Leben bezwecken, vornehmlich auf die Ermöglichung (beispielsweise berufliche Ausbildung), Maßnahmen, die der Verhinderung von Dissoziation dienen, vornehmlich der Erhaltung, und Maßnahmen, die auf Resozialisierung ausgerichtet sind, vornehmlich auf die Wiederherstellung subjektiver Teilnahmekompetenzen. Handlungszusammenhänge, die auf die Entwicklung bzw. Sicherung der Teilnahmekompetenzen in ihren drei Varianten verpflichtet sind, können auch als pädagogische Handlungszusammenhänge bezeichnet werden. Ihr Arrangement, ihre Realisierung und Kultivierung sind gesellschaftlich dem Bildungsbereich und seiner Reflexionsinstanz, der Erziehungswissenschaft überantwortet. In diesem Sinne ist die Förderung von Future Skills durch den Bildungsbereich auf die Entwicklung und Sicherung der Partizipation von Individuen am gesellschaftlichen System ausgerichtet.

Bildung in einem solchen Sinne wird strukturell als ein dreifaches Verhältnis des Einzelnen zur dinglichen Welt, zur Gesellschaft und zu sich selbst verstanden (Meder 2007: 199ff; Meder 2000: 36f.; ausführlich dazu auch 1999: 25ff.). Dieser Bildungsbegriff macht klar, dass Bildung als Forschungsgegenstand nicht ein Substrat oder eine Substanz ist, sondern eine Relation - also ein Verhältnis bzw. eine Beziehung. Diese dreigeteilte Struktur ist im vorliegenden Future Skills Konzept übernommen worden, indem das Future Skills Konzept Kompetenzfelder für alle drei Bereiche enthält. Was am Einzelnen erkannt werden kann, ergibt sich dabei aus der Relation, bzw. dem Verhältnis, das als Verhalten bezeichnet werden kann (Meder 2007). Bildung fungiert als Prozess der Ausbildung der genannten Verhältnisse. In seiner strukturellen Fassung liefert dieser Bildungsbegriff allerdings nur die Perspektive einer pädagogischen Analyse, aber noch kein Kriterium für die Entscheidung, ob ein Fall für ein Eingreifen vorliegt, das heißt, ob ein Fall dafür gegeben ist, dass ein pädagogisches Handlungsarrangement auf ein bestimmtes Ziel hin realisiert werden soll oder muss. Um diese Entscheidung zu fällen, bedarf es Kriterien, Normen und/ oder Werten. Von daher muss der Bildungsbegriff als Konzept des dreifachen Verhältnisses normativ aufgeladen werden, damit klar ist, wie das richtige Verhältnis zur Welt, zur Gesellschaft und zu sich selbst aussieht. Denn nur so kann entschieden werden, ob ein Fall für professionelles Eingreifen vorliegt.

Darüber hinaus bedarf es einer weiteren normativen Orientierung. Hat man beispielsweise professionell diagnostiziert, dass das vorliegende Verhältnis zu sich selbst keine Partizipation erlaubt und damit ein Eingreifen seitens des Bildungs- und Erziehungssystems notwendig wird, dann besteht noch immer keine Orientierung darüber, was zu tun ist, um den unerwünschten Ist-Zustand in einen gesellschaftlich und individuell verantwortbaren Soll-Zustand zu transformieren. Es fehlt also zur Umsetzung eine Norm, die zeigt was qualitativ sinnvolles professionelles 
Handeln im pädagogischen Feld ist. Ein solches Handlungswissen ist, neben hermeneutischem und alltagsbezogenem Wissen, eine Grundbedingung für jede Art von Professionalität. Das Future Skills Konzept ist in diesem Sinne eine normative Ausgestaltung des Ziels Sicherung der Partizipation, im Sinne einer pädagogischen Vermittlung von Teilnahmekompetenzen.

\section{B 2.4 Emergenz und Selbstorganisation}

Emergenz ist wie die neue Folie, auf der sich die Entwicklung von Organisationen, Abläufen und dem sozialen Miteinander in modernen Gesellschaften vollzieht. Sie ist gewissermaßen der Schlüssel zum Verständnis von Systemen und ihrer Eigenschaften. Emergenz gibt Auskunft darüber, ob und auf welcher Regelbasis Selbstorganisation in sozialen Systemen funktioniert. Sind Abläufe nicht mehr vorgegeben oder regelbasiert, stellt sich die Frage, ob es andere Gesetzmäßigkeiten als die bisher bekannten gibt, die es möglich machen, Entwicklungen abzusehen und zu verstehen. Dazu liefert Emergenz als Konzept die Grundlage.

Der springende Punkt besteht darin, dass emergente Eigenschaften eines Systems sich nicht - oder jedenfalls nicht offensichtlich - auf Eigenschaften der einzelnen Elemente des Systems zurückführen lassen, die diese isoliert aufweisen. So wird beispielsweise im Bereich der Gehirnforschung und in der Philosophie des Geistes von einigen Wissenschaftlerinnen und Wissenschaftlern die Meinung vertreten, dass das Bewusstsein eine emergente Eigenschaft des Gehirns sei (Stephan 2016).

Stephan (ebenda) führt aus, dass emergente Phänomene in der Physik, Chemie, Biologie, Mathematik, Psychologie oder Soziologie beschrieben werden. Damit würden Emergenztheoretiker deutlich bestreiten, dass eine vollständige Beschreibung der Welt allein aufgrund der Kenntnis der Elementarteilchen und allgemeiner physikalischer Gesetze prinzipiell möglich ist. Die Anerkennung emergenter Phänomene muss aber nicht zu einem Verzicht auf wissenschaftliche Erklärung führen. Vielmehr zeigen die Entwicklungen der Synergetik, Systemtheorie und der Chaosforschung, dass emergenzverwandte Phänomene wie Selbstorganisation und ihre Entstehungsbedingungen durchaus systematischen und auch objektiv nachvollziehbaren Erklärungen zugänglich sind (siehe dazu auch Greve \& Schnabel 2011). Allerdings tritt an die Stelle der Einheit der Wissenschaft aufgrund einer hierarchischen Ableitung aus universalen Gesetzen, ein transdisziplinärer Dialog, dessen Ziel es ist, analoge Strukturen komplexer Systeme auf unterschiedlichen Emergenzebenen zu vergleichen. Emergenz entsteht in den meisten Fällen auf Basis spontaner Selbstorganisation. Man versteht unter Emergenz das Auftauchen von 
Systemzuständen, die nicht durch die Eigenschaften der beteiligten Systemelemente erklärt werden können. In gewissem Sinne entstehen auf höheren Stufen neu auftauchende Qualitäten aus vorherigen Zuständen. Dabei ist zu beachten, dass die neu auftauchenden Qualitäten erst entstehen müssen und nicht bereits vorhanden sind. Im Volksmund wird es so ausgedrückt: Das Ganze ist mehr als die Summe seiner Teile. Für dieses Mehr bzw. für dessen Entstehung steht der Begriff der Emergenz.

Das Phänomen der Emergenz kann am Beispiel der Temperatur verdeutlicht werden. Betrachtet man ein einzelnes chemisches Molekül wie z.B. das Wassermolekül, dann kann man für dieses Molekül keine Temperatur bestimmen. Hat man allerdings eine große Menge des einzelnen Moleküls, dann ist es möglich eine Temperatur zu ermitteln. Die Temperatur entsteht erst, wenn viele Moleküle aufeinandertreffen, somit kann die Temperatur als eine emergente Eigenschaft vieler Moleküle angesehen werden. So ist die Temperatur des Wassers eine emergente Eigenschaft der Wassermoleküle.

Nach Stephan (ebenda, auch Stein 2004) beschreibt Emergenz systemisch ausgedrückt einen spezifischen Transformationsprozess zwischen zwei Systemzuständen. Besitzt ein System den aktuellen Systemzustand A und wird dieses System in einen neuen Systemzustand B überführt, so findet eine Transformation von Systemzustand A nach Systemzustand B statt. Die Transformation ist das Ergebnis eines Transformationsprozesses. Man bezeichnet den Transformationsprozess als emergent, wenn der Systemzustand B sich nicht direkt aus Systemzustand A und seinen Teilchen oder seinen Teilsystemen ergibt (Stein 2004). Diese Betrachtung von Emergenz im Rahmen eines Transformationsprozesses trägt auch zur wissenschaftlichen Klärung des Konzeptes bei. Denn es kann nun gefragt werden, welche Transformationsregeln eigentlich wirken. Sind keine Transformationsregeln zu erkennen oder bekannt, würde man auch nicht mehr von Emergenz sprechen. Während des Transformationsprozesses treten neue Qualitäten auf, die nicht auf die Aufsummierung der Einzeleigenschaften zurückgeführt werden können.

Daraus ergibt sich die Frage, ob das Emergenzphänomen sich überhaupt auf einfache Transformationsregeln reduzieren lässt. Zwei Prinzipien stehen bei Emergenz im Mittelpunkt:

- Prinzip 1 - Irreduzibilität: der neue Zustand eines Systems lässt sich nicht (historisch) auf den alten linear zurück reduzieren, sondern stellt einen qualitativ neuen Zustand dar.

- Prinzip 2 - Unvorhersagbarkeit: weder zeitlich noch inhaltlich kann vorhergesagt werden, in welchen Zustand das neue System sich transformiert.

Im Folgenden geht es nun weiter um den Tranformationsprozess. Wie vollzieht er sich - welche Erklärungsmodelle für die Transformation gibt es, welche Regeln 
wirken und ist eine Systematik zu erkennen? Diesen Fragen wollen wir uns im Folgenden im Detail zuwenden. Im Mittelpunkt des Transformationsprozesses steht das Phänomen der Selbstorganisation, die für die Erklärung des Emergenzphänomens die wesentliche Rolle spielt.

Die modernen Selbstorganisationstheorien stammen aus Physik und Biologie und durchdringen das wissenschaftliche Denken mehr und mehr. Sie bilden die Grundlage für die Entstehung neuer Bedarfe des Arbeitsmarktes, die wir in diesem Buch als Future Skills bezeichnen. Wir wollen die großen Bereiche von Emergenz, Selbstorganisation, Synergetik und mehr oder weniger radikalem Konstruktivismus nicht in Gänze vorstellen. Stattdessen möchten wir uns im Folgenden auf einige begrenzte Beispiele aus dem Bereich der Synergetik, dem Ökosystemansatz, der Medientheorie und der Autopoiesis konzentrieren.

\section{B 2.5 Synergetik und Selbstorganisation}

Als erstes Erklärungsmodell wird die Wissenschaftsdisziplin Synergetik beschrieben. Synergetik ist die Lehre vom Zusammenwirken (Haken 1991: 17). Sie wurde in den sechziger Jahren von Herrmann Haken, einem Stuttgarter Physiker, entwickelt. $\mathrm{Zu}$ dieser Zeit entdeckte er die Lasertechnik. Dabei war von Interesse, warum sich die an einer diffusen Lichtquelle ausgestrahlten verschiedenen Lichtwellen zu einer Lichtwelle bündeln und sich dadurch der Laserstrahl bildet. Es stellte sich die Frage, warum es bei verschiedenen Lichtwellen zu einem Selbstorganisationsprozess mit dem Ergebnis einer einzigen Lichtwelle kommt. In dieser Frage ist auch die Definition des Begriffes Selbstorganisation angesprochen. Stein (2004) führt hierzu aus, dass man in einem System von Selbstorganisation spricht, wenn ein Systemzustand einzig von den Systemelementen und den Relationen zwischen ihnen hervorgerufen wird, ohne Einfluss der Umwelt auf das System. Die Synergetik versteht sich als eine fachübergreifende Wissenschaftsdisziplin, ähnlich der Mathematik und Statistik (ebenda). Haken (1991) betont, dass die Synergetik nicht nur auf die Naturwissenschaft angewendet werden kann, sondern eine Anwendung z. B. in Gesellschaftswissenschaften wie der Soziologie ebenfalls möglich ist. Die Synergetik kann verstanden werden als eine Lehre des Zusammenwirkens und als ein Konzept zur Erklärung von Ordnungsbildung in Systemen mit vielen interagierenden Einheiten. Haken untersucht mit der Synergetik, wie sich eine große Anzahl von Einzelelementen zu höheren Strukturen selbst organisiert. John Erpenbeck und Volker Heyse (1999) geben hierzu in Anlehnung an den Physiker Hermann Haken folgendes Beispiel: 


\begin{abstract}
„Denken wir uns ein Schwimmbad, bei dem die Schwimmerinnen und Schwimmer in einer Richtung zum anderen Rand und zurückschwimmen sollen. Ist das Schwimmbecken sehr voll, wie das an heißen Sommertagen der Fall ist, so sind sehr viele Schwimmerinnen und Schwimmer unterwegs und behindern sich beim Hinund Herschwimmen. Deshalb kommen manche Bademeisterinnen und Bademeister auf die Idee, die Schwimmenden im Kreis herumziehen zu lassen. Die gegenseitige Behinderung ist dabei viel kleiner. Hier ist den Schwimmerinnen und Schwimmern vom Badepersonal eine kollektive Bewegung vorgeschrieben worden. Aber auch ohne Bademeisterin oder Bademeister können die Schwimmerinnen und Schwimmer auf die Idee kommen, im Kreis zu schwimmen. Erst sind es vielleicht nur einige, aber immer mehr schließen sich ihnen an, da die Kreisbahn auch für diese bequemer ist. So entsteht schließlich eine kollektive Bewegung und zwar ohne äußere Anordnung, das heißt selbstorganisiert." (Haken \& Portugali 1995)
\end{abstract}

Es stellt sich also selbstorganisiert ein Ordnungszustand oder kurz Ordner ein. Niemand steht draußen am Rand und ruft ordnend, normierend: Jetzt schwimmen wir mal alle im Kreis, links oder rechts herum! Im Gewusel der Schwimmerinnen und Schwimmer schwimmen einige eher zufällig in eine Richtung, nach links oder rechts. Diese Instabilität setzt sich schnell, fast schlagartig durch, zwingt alle, die sich noch ungeordnet bewegen, auf die Kreisbahn. Die Kreisbahn bildet einen Ordner. Der Ordner, im skizzierten Beispiel, die Kreisbewegung und die von ihm versklavten Teile, die Schwimmenden, bedingen sich in ihren Bewegungsformen gegenseitig.

„Durch die Kollektivbewegung der Teile entsteht der Ordner. Der Ordner umgekehrt "versklavt" die Teile, indem er sie in den Ordnungszustand zwingt.“" (Haken \& Portugali 1995)

Haken formuliert, dass sich durch die „Versklavung“ der Individuen, durch den Ordner ein Phasenübergang bildet. Während des Phasenübergangs zeigen sich bereits Eigenschaften von beiden Phasen, der alten und der neuen. Allerdings besteht keine Kausalität zwischen den Phasen. Es kann nicht vorhergesagt werden, welcher neue Zustand durch den Ordner hervorgerufen wird.

Ein anderes Beispiel:

„Eine Treppe mit Fußgänger(innen)verkehr in Deutschland. Es ist sehr wahrscheinlich, dass sich „Rechtsverkehr" ergibt, allerdings ist das nicht zwangsläufig. Schon wenige englische Touristinnen und Touristen auf einer Treppe reichen aus, um vielleicht einen Ordner für „Linksverkehr“ zu bilden. (Haken \& Portugali 1995)

Haken (1991) versteht unter „Nichtlinearität“, dass kleinste Änderungen der Systemstruktur riesige Auswirkungen auf den Systemzustand haben können. Durch 
die Ordner findet eine Komplexitätsreduzierung statt. Es ist nicht nötig, das genaue Verhalten der einzelnen Individuen zu kennen, es reicht zu wissen, welche Ordner für die Individuen maßgebend sind (Haken 1991: 23). Als Beispiel führt Haken die Erbsubstanz DNS (Desoxyribonukleinsäure) der Lebewesen an. Trotz des riesigen Umfangs der DNS ist in diesem nicht die Information für jede einzelne Körperzelle abgelegt. Vielmehr enthält die DNS lediglich Informationen für die verschiedenen Zelltypen sowie die Information zur Bildung von Ordnern, die für eine Strukturierung der Zellen sorgen. Während der Selbstorganisation kann es passieren, dass mehrere Zustände nach dem Phasenübergang gleichwahrscheinlich sind. In dieser Situation entscheidet der Zufall, welcher Zustand sich nach dem Phasenübergang ergibt. Daraus folgt, dass eine Vorhersagbarkeit nicht möglich ist. Das System neigt zum Nichtdeterminismus (Erpenbeck \& Heyse 1999).

Damit es überhaupt zu einem Phasenübergang kommt, muss dem System Energie zugeführt werden. In sozialen Systemen tritt an die Stelle der Energie die Information. Bevor wir auf die besondere Bedeutung von Informationen als induzierende Momente für den Phasenzustandswechsel sozialer Systeme und die Digitalisierung eingehen, noch einmal zu den Grundprinzipien von Selbstorganisation in der Theorie Hakens.

Nach Mainzer (1992) können selbstorganisierte Systeme prinzipiell nicht vollständig von außen gelenkt und gesteuert werden. Sie unterliegen innerer Bedingtheit und Bestimmtheit. Ihre Strukturen sind demnach vor allem durch innere Faktoren bedingt. Ihre Zukunft ist real, offen. Erpenbeck (2018) beschreibt die Übertragung von Hakens Selbstorganisationstheorie auf den Prozess menschlicher Handlungen und Wertungen und benennt wichtige Prinzipien für selbstorganisierte Systeme, die einen wichtigen Hintergrund für die Entwicklung von Future Skills bilden:

1. Er führt aus, dass erstens in allen solchen Systemen, das bereits beschriebene Prinzip des Ordnungsparameters gilt. Das heißt, es existieren in der Regel spezielle Bewegungen, die alle Teilbewegungen koordinieren, konsensualisieren, manchmal auch versklaven. Dies gilt im übertragenen Sinne auch für geistiges und symbolisches Handeln, das durch übergeordnete Ordnungsparameter, nämlich Werte und Normen, koordiniert wird. Die Entstehung derartiger Ordner ist kaum zu prognostizieren und schwer zu administrieren.

2. Er führt zweitens aus, dass für alle selbstorganisierten Systeme nur eine beschränkte Vorhersagbarkeit gilt. Ihre Entwicklungen lassen sich prinzipiell nicht sehr langfristig, manchmal nicht einmal kurzfristig, prognostizieren. Vielmehr gilt das Prinzip der Historizität. Durch Entwicklung und Evolution entstandene Strukturen und Prozesse lassen sich nur im Kontext ihrer konkreten Entstehungsgeschichte verstehen. 
3. Das Prinzip der Komplexität ist drittens für soziale, selbstorganisierte Systeme wichtig. Schon aufgrund ihrer Komplexität sind die meisten Systeme nur unvollständig beschreibbar. Innere Zustände beeinflussen sich selbst. Das Systemverhalten ist weder aus Inputs noch aus internen Zuständen ableitbar. Die Komplexität ist nicht reduzierbar.

4. Viertens gilt das Prinzip der Redundanz. Information ist über das System verteilt. Es gibt kein ausschließliches Hierarchieprinzip. Die Gestaltung und Lenkung des Systems kann aus Teilsystemen heraus erfolgen. Unterschiedliche Werte mit analogen Funktionen, aber auch analoge Werte mit unterschiedlichen Funktionen können entstehen, friedlich nebeneinander existieren, sich aber auch heftig bekriegen.

5. Es ist fünftens das Prinzip der Selbstbezüglichkeit, der Selbstreferentialität von selbstorganisierten Systemen zu beachten. Ihr Systemverhalten ist das Produkt eines inneren Zusammenhangs. Jedes Handeln wirkt auf das System selbst zurück und ist Ausgangspunkt weiteren Handelns.

6. Sechstens das Prinzip der Autonomie. Das selbstorganisierte System ist zwar nicht informationell unabhängig, aber im Sinne von Selbstgestaltung, -lenkung und Entwicklung selbstbestimmt gegenüber der Umwelt.

7. Und siebtens und letztens weist Erpenbeck darauf hin, dass soziale Systeme immer selbstorganisiert und kreativ sind; immer werte- und willensgesteuert, sinnund zweckorientiert, sie beruhen auf Kommunikation, Symbolen und Lernen.

Die Wichtigkeit von Informationen für die Änderung des Phasenzustands von sozialen Systemen, ihr Einfluss und die Bedeutung der Digitalisierung wurde u. a. durch Dirk Bäcker (2018), Professor für Soziologie an der Universität Witten Herdecke aufgearbeitet und wird in Kapitel B 2.7 Digitalisierung und Selbstorganisation näher beschrieben.

\section{B 2.6 Ko-Evolution und Selbstorganisation: Ökosystemtheoretische und sozialökologische Erklärungsansätze}

Urie Bronfenbrenner gründete 1978 eine ökologische Sozialisationsforschung, die sich ähnlich der qualitativen Sozialforschung für natürliche Alltagssituationen der Menschen und deren subjektiven Sinngebungen interessierte. André Epp (2018) bewertet dies auch als eine Kritik an den vorherrschenden psychologischen Laborexperimenten der siebziger Jahre und den damit verbundenen determinis- 
tischen Theorien hat der Psychologe. Er veröffentlichte dabei das ökosystemische Entwicklungsmodell, in das er sowohl die ursprüngliche soziale als auch die biologische Bedeutung des Begriffes Ökologie aufnahm (Bronfenbrenner 1976). Erstere Bedeutung leitet sich vom griechischen Wort oikos (griechisch für Haushalt bzw. Hausgemeinschaft) ab, verweist also auf die Art und Weise, wie der Haushalt zusammengesetzt, die Familie organisiert ist und wie diese zu anderen Menschen in Beziehung steht. Ihre Bedeutung ist an biologische Ökosysteme angelehnt. Diese bestehen aus biotischen Gemeinschaften von aufeinander bezogenen Organismen, die sich zusammen den gleichen Lebensraum teilen. Zu berücksichtigen ist, dass Ökosysteme unterschiedliche Größen haben und sich wechselseitig überschneiden können (Epp 2018). Menschliche Ökosysteme umfassen jedoch nicht nur biologische, sondern auch kulturelle Lebensbedingungen.

Bronfenbrenner (1981) verweist mit seinem Modell darauf, dass Entwicklung als ein wechselseitiger interaktionistischer Prozess zwischen dem Individuum und seiner sozialen Umwelt betrachtet werden muss. Dabei sind die Interaktionen ineinander verschachtelt und die verschiedenen Elemente des Systems beeinflussen sich wechselseitig. Die Veränderung eines Elements kann die Modifikation anderer nach sich ziehen (Oerter 1995: 88), sodass sich ein Geflecht von Interaktion und Beziehung herausbildet. Der ökologische Übergang ist somit immer Folge wie auch Anstoß von Entwicklungsprozessen, die sowohl positiv als auch negativ sein können.

Heute findet der Begriff des Ökosystems immer öfter auch im Zusammenhang mit Organisationen und wirtschaftlichen Beziehungsnetzwerken Verwendung. Im Jahre 1989 transferierten Robert A. Frosch und Nicholas E. Gallopoulos (1989) das Konzept zunächst in den Bereich industrieller Ökosysteme. Micheal Rothschild wiederum bezeichnete ein Jahr später gar die ganze (kapitalistische) Wirtschaft als „living ecosystem“ (später veröffentlicht in Rothschild 2004). Der wissenschaftliche Durchbruch gelang im Jahr 1993, als James F. Moore das Konzept der Business Ecosystems im Harvard Business Review veröffentlichte und die Inhalte in seinem Buch The Death of Competition verfeinerte (Moore 1996). Moore spricht darin von der sich im Laufe der Zeit entwickelnden Koevolution verschiedener Organismen des Business Ecosystems, die sich zunehmend an den Richtungsvorgaben der im Ecosystem führenden Parteien orientieren:

\footnotetext{
"An economic community supported by a foundation of interacting organizations and individuals - the organisms of the business world. The economic community produces goods and services of value to customers, who are themselves members of the ecosystem. The member organisms also include suppliers, lead producers, competitors, and other stakeholders. Over time, they coevolve their capabilities and roles, and tend to align themselves with the directions set by one or more central companies. Those companies holding leadership roles may change over time, but the
} 
function of ecosystem leader is valued by the community because it enables members to move toward shared visions, to align their investments, and to find mutually supportive roles." (Moore 1996)

Der ökologische Übergang kann auch als ein Phasenübergang von Systemen im Emergenzprozess beschrieben werden, wie sie oben dargelegt wurden. Veränderungen betreffen somit grundsätzlich nicht nur die individuelle Ebene, sondern das ökologische System als Ganzes. Unter Ökologie wird die Gesamtheit der potentiellen und rezipierten Umweltbedingungen eines Individuums gefasst, so wie die Transkation, also die Aktivität und Dynamik im gesamten System zwischen dem Individuum und seiner Umwelt (Epp 2018). Folglich finden nicht nur die Interaktionen im unmittelbaren Lebensraum eine Berücksichtigung, sondern auch zunächst entfernter erscheinende Kontexte wie beispielsweise strukturelle oder normative Bedingungen des Gesellschaftssystems rücken in das Blickfeld, da Menschen von diesen beeinflusst werden, sie diese aber andererseits wiederrum selbst beeinflussen (Seifert 2011: 114 in Epp 2018). Bronfenbrenner bezeichnet diese Strukturen als Mikro-, Meso-, Exo- und Makrosysteme, wobei jedes folgende größer und umfassender als das vorausgegangene ist (Oerter 1995: 88).

- Unter dem Mikrosystem werden all diejenigen Faktoren gefasst, die dem Individuum in seinem Handeln durch ein anderes Individuum zugeschrieben werden; also bestimmte äußerliche Merkmale, Fähigkeiten usw. Es handelt sich um personale Einflussgrößen, die im Individuum verortet werden.

- Epp führt aus, dass das Mesosystem die Wechselbeziehung zwischen den Lebensbereichen umfasst, an denen sich die entwickelnde Person aktiv beteiligt, wie ein Kind etwa die Beziehung zwischen Elternhaus, Schule und Kameradengruppe, für einen Erwachsenen die zwischen Familie, Arbeit und Bekanntenkreis (Bronfenbrenner 1981: 41). Zum Mesosystem gehören dementsprechend die verschiedenen Lebenskontexte, in denen sich ein Individuum bewegt, was auch die Organisationen einschließt.

- Diejenigen Bereiche, an denen sich die entwickelte Person nicht selbst beteiligt, in denen aber Ereignisse stattfinden, die beeinflussen was in ihrem Lebensbereich geschieht, werden als Exosystem bezeichnet (Bronfenbrenner 1981: 42). Dies beinhaltet formelle und informelle Strukturen, zu denen das zu entwickelnde Individuum nicht unmittelbar als handelnde Person gehört. Es ist dort also nicht anwesend. Stattdessen beeinflussen diese Strukturen indirekt das Individuum. Andererseits beeinflusst das Individuum aber auch auf Umwegen diese Strukturen. Zusammengefasst können Exosysteme als Quellen von Effekten aus entfernten Umweltregionen beschrieben werden. Darunter fallen die größeren 
Institutionen der Gesellschaft und wie sich diese auf der konkreten lokalen Ebene entfalten.

- Interessant ist, dass der Begriff des Makrosystems sich nicht auf spezifische Kontexte wie das Leben des oder der Einzelnen richtet und diese betrifft, sondern vielmehr auf übergeordnete institutionelle Muster, Strukturen und Aktivitäten. Epp (2018) definiert mit Bronfenbrenner, dass das Makrosystem sich auf die grundsätzliche formale und inhaltliche Ähnlichkeit der Systeme niedrigerer Ordnung bezieht, die in der Subkultur oder in der ganzen Kultur bestehen oder bestehen können, einschließlich der ihnen zugrundeliegenden Weltanschauung und Ideologien. Beispiele hierfür sind das politische System, das soziale, juristische System und globale übernationale Organisationen und Institutionen.

Epp (ebenda) führt weiterhin aus, dass grundsätzlich gelte, dass es sich bei den unterschiedlichen Systemebenen um eine topologisch ineinander geschachtelte Anordnung handelt, die jeweils als ein die nächste Struktur umschließendes Gebilde verstanden werden kann. Dementsprechend wirkt die Makroebene nicht direkt auf die Mikroebene, sondern die Wechselwirkung der einzelnen Ebenen und in ihnen enthaltenen Systeme muss bedacht werden. Da Veränderungen im ökosystemischen Entwicklungsmodell grundsätzlich als ein Konglomerat miteinander interagierender und kommunizierender Systeme und Faktoren verstanden werden, werden Parallelen zum symbolischen Interaktionismus deutlich. Bronfenbrenner teilt implizit dessen wissenschaftstheoretische Annahmen. Mittels des ökosystemischen Entwicklungsmodells können die Relevanzstrukturen und Realitätsebenen rekonstruiert und analysiert werden, inwiefern Menschen in unterschiedlichen gesellschaftlichen Kontexten (Mikro-, Meso-, Exo- und Makrosysteme) Bedeutung zuschreiben und welche Rolle diese für die Konstruktion ihrer Realität besitzen (Epp 2018). Da Wirklichkeit nicht als vorgegeben erachtet wird, sondern durch Subjekte kontinuierlich konstruiert wird, erhalten soziale bzw. gesellschaftliche Systeme ihre Bedeutung erst durch die Interpretationsleistungen der Handelnden.

Auf diesen Annahmen baut auch der sozialökologische Ansatz Dieter Baackes (1980) auf. Sozialökologische Ansätze untersuchen die Wechselbeziehungen zwischen sozialer Umwelt und sozialem Verhalten des Menschen (Ehlers 2011). Sozialisation wird dabei verstanden als Folge aktiver Prozesse der Auseinandersetzung mit der symbolischen, sozialen und materiellen Umwelt sowie sich selbst. In die pädagogische Jugendforschung in Deutschland hat Dieter Baacke den sozialökologische(n) Ansatz zur Beschreibung und Erklärung des Verhaltens Jugendlicher im Anschluss an Bronfenbrenner eingebracht (Baacke 1980; Bronfenbrenner 1974, 1976). In den folgenden Jahren wurde er von der Arbeitsgruppe um Dieter Baacke zunächst in 
der Jugendforschung (Sander \& Vollbrecht 1985), später auch als Mediensozialisationsansatz empirisch umgesetzt (Baacke 1988; Baacke, Sander \& Vollbrecht 1988; Baacke, Frank \& Radde 1991). Als besonders ertragreich erwies sich das Projekt Medienwelten Jugendlicher (Baacke, Sander \&Vollbrecht 1990a und 1990b), mit zahlreichen Publikationen in unterschiedlicher Autorenschaft (Baacke, Sander \& Vollbrecht 1988; Vollbrecht 1988; Vollbrecht 1990; Treumann et al. 2002).

Mit Bennewitz kann man formulieren: Die soziale Welt wird als eine durch interaktives Handeln konstruierte Welt verstanden, die für den einzelnen aber auch Gruppenkollektive sinnhaft strukturiert ist. Soziale Wirklichkeit stellt sich somit als Ergebnis von sozial sinnhaften Interaktionsprozessen dar (Bennewitz 2010: 45). Die Ökosystemtheorie bietet also einen Erklärungsansatz, der darstellt, in welcher Weise soziale Systeme und Individuen auf unterschiedlichen Ebenen vom Individuum bis zur globalen Sozialstruktur zusammenwirken. Dirk Bäckers Medienanalyse zeigt, wie Medien diese unterschiedlichen Ebenen beeinflussen und zusammenbringen und Überschuss an Sinn und an Informationen dazu führt, dass in den jeweiligen Teilsystemen sich gegenseitig beeinflussende Selbstorganisationsprozesse ablaufen. Wie diese Selbstorganisationsprozesse funktionieren, erklärt Herrmann Hakens Theorie in der Synergetik.

Mit dem Ansatz der Autopoiesis können Selbstorganisationsprozesse ebenfalls erklärt und konzeptualisiert werden. Das Konzept der Autopoiesis ist eine Teilmenge des allgemeingültigen ontologischen Konzepts der emergenten Selbstorganisation. In der Biologie stellt das Konzept der Autopoiesis einen Versuch dar, das charakteristische Organisationsmerkmal von Lebewesen oder lebenden Systemen mit den Mitteln der Systemtheorie zu definieren. Der vom chilenischen Neurobiologen Humberto Maturana (1987) geprägte Begriff wurde in der Folge seiner Veröffentlichungen aufgebrochen und für verschiedene andere Gebiete wissenschaftlichen Schaffens abgewandelt und fruchtbar gemacht und soll im Folgenden im Kontext der Selbstorganisation thematisiert werden.

\section{B 2.7 Digitalisierung und Selbstorganisation}

In sozialen Systemen kommt es durch das Entstehen von Ordnern zu innerer Strukturbildung, die als Phasenübergang bezeichnet werden kann. Dabei kann dieser Phasenübergang unter Bedingung der Energiezufuhr bei natürlichen Systemen und Informationszufuhr bei sozialen Systemen ausgelöst werden. Die Digitalisierung fungiert als Medium, welches einen Informationsüberfluss für alle sozialen Systeme darstellt (Bäcker 2018). Die Aufarbeitung der gesellschaftlichen Entwick- 
lung anhand der sogenannten Archäologie der Medienepochen von Dirk Bäcker, einem Soziologen an der Universität Witten-Herdecke, macht dies deutlich. Seine Hypothese ist, dass elektronische Medien der Gesellschaft an der Schnittstelle von Mensch und Maschine einen „Überschusssinn“ (Bäcker 2018) bereitstellen, auf dessen Bearbeitung bisherige Formen der Gesellschaft strukturell und kulturell nicht vorbereitet sind. Es werden also durch die Bereitstellung eines Überschusses an Sinn und Informationen durch elektronische Medien, Anpassungs- und Kompensationsbewegungen in gesellschaftlichen Systemen ausgelöst, deren Gestaltungs- und Strukturierungsrichtung nicht vorhersehbar ist und im Sinne von Emergenz zu selbstorganisierten Prozessen führt.

Die Idee des Begriffes des „Überschusssinns“ folgt einem Vorschlag von Niklas Luhmann (1997: 405), verschiedene Formen der Gesellschaft unter dem Gesichtspunkt jeweils dominanter Verbreitungsmedien der Kommunikation zu beobachten und in diesem Sinne zwischen der tribalen, der antiken, der modernen und einer nächsten Gesellschaft (next society) zu unterscheiden. In diesen sind zunächst a) die Sprache, dann b) die Schrift, dann c) der Buchdruck und schließlich die elektronischen Medien jeweils dominant. Dabei ist zu erkennen, dass jedes in der Evolution der Gesellschaft neu auftretende Verbreitungsmedium neue Möglichkeiten der Kommunikation in sich trägt und ausprägt, mit denen neue, bisher unverbundene Akteurinnen und Akteure auf neue Weise miteinander in Kontakt treten. Laut Bäcker bedroht das Erreichen und Verstehen neuer Kreise von Adressaten die bisherige Struktur und Kultur, bringt sie in die Instabilität und Imbalance und destabilisiert die bisherigen Institutionen, Konventionen und Routinen, die auf die Modalitäten der älteren (Verbreitungs-)Medien eingestellt sind. Genau in dieser Instabilität liegt nun der Moment der Selbstorganisation des Neuen in sozialen Systemen und Organisationen.

Bäcker (2018) führt weiter aus, dass die Sprache einen Überschusssinn produziert, der über die Wahrnehmung von Körpern, Gesten, Bewegungen und allenfalls einigen Warn- und Trostlauten hinausgeht und die Menschheit mit dem Drama konfrontiert, zwischen Wort und Sache unterscheiden lernen zu müssen, um eine Sprache (inklusive ihrer Möglichkeit der Lüge) überhaupt handhaben zu können. Der Bewältigung des Referenzproblems der Sprache (Deacon 1997) inklusive der Einführung von Moral und Geheimnis zur Kontrolle der Frage „wer mit wem worüber reden darf" und zur Markierung dessen, worüber nicht gesprochen werden darf, verdanke die tribale Gesellschaft ihre Entstehung (Luhmann 1997: 230 nach Bäcker 2018). In eine weitere Medienepoche tritt die Menschheit in dem Moment ein, so Bäcker weiter, in dem zunächst die Schrift und dann die alphabetische Schrift einen neuen Überschusssinn produzieren, indem sie die Zeithorizonte der Gesellschaft explodieren lassen. Die Schrift ermöglicht kontrollierbare Zugriffe 
auf eine differenzierbare Vergangenheit und korrigierbare Zugriffe auf eine noch offene Zukunft. Schriftgesellschaften sind deswegen historische und wegen ihres reflexiven, das heißt laufend überprüften Umgangs mit Mythen, sog. „heiße Gesellschaften" (Lévi-Strauss 1962). Die Begriffe heiße und kalte Gesellschaften bzw. Kulturen, gehen auf das Werk „Das wilde Denken“ des französischen Ethnologen Claude Lévi-Strauss aus dem Jahr 1962 zurück. In diesem unterscheidet er Kulturen nach ihrer weltanschaulichen Einstellung zum Kulturwandel. Je kälter eine Gesellschaft auf der Skala demnach ist, desto ausgeprägter ist ihr Bestreben, ihre traditionellen Kulturmerkmale möglichst unverändert zu bewahren - eine Kultur wird dagegen als umso heißer eingeordnet, je größer ihr Antrieb zu tiefgreifenden und schnellen Modernisierungen der Gesellschaft ist. Die Schrift erschließt als lineare und offene Perspektiven, eine Vergangenheit und eine Zukunft, die zuvor in der ewigen Wiederkehr der Erinnerung an die Ahnen zirkulär verschlossen war. Die in einem variierbaren Gedächtnis und in variierbaren Plänen enthaltene Komplexität der Gesellschaft wird durch Stratifikation aufgefangen, die es erlaubt, unterschiedlichen Sozialschichten die Orientierung an unterschiedlichen Zeithorizonten zuzuordnen.

Jede dieser Medienepochen ist durch einen Überschusssinn gekennzeichnet, der die vorherige Ordnung bedroht und nur in einer neuen Ordnung aufgefangen werden kann. Andernfalls müsste die Gesellschaft Mittel und Wege finden, das jeweilige neue Verbreitungsmedium der Kommunikation abzulehnen. Tatsächlich begleitet der Versuch der Ablehnung die Einführung jedes neuen Verbreitungsmediums. Seit der Einführung der Schrift gibt es dafür Beispiele in Hülle und Fülle. Dass Kommunikation entkörpert, gilt nicht erst seit der Einführung und Durchsetzung der neuen elektronischen Kommunikationsmedien oder des Buchdruckes, sondern seit der Einführung der Schrift und bereits der Sprache, auch wenn die Reaktion der Gesellschaft auf die Sprache aus naheliegenden Gründen nicht dokumentiert ist. Die Ablehnung der neu entstehenden Medien - so Bäcker (2018) - ist ein Topos, der medien- und kulturkritisch bis heute wiederholt wird. Entscheidend sei jedoch, dass die Ablehnung neu auftretender Medien ihrerseits eine Form der Beobachtung ihrer möglichen Konsequenzen und damit eine Form der Entdeckung möglichen Nutzens ist - auch wenn man diesen nur dadurch realisieren kann, indem man die Ablehnung überwindet und gegen Strukturen der Gesellschaft verstößt. Die Medienevolution der Gesellschaft findet im Medium der Ablehnung von Medieninnovationen statt.

Jedes Medium ist daher zum Zeitpunkt seines Auftretens auch als disruptiv zu bewerten. Die dann jeweils von Ökonomen nachgewiesene Senkung der Transaktionskosten überzeugt immer nur die einen und bedroht die anderen, deren Renten von einer Ausbeutung der Transaktionskosten abhängen. Es hängt von 
technischen - ebenso wie sozial - findigen Innovationen ab, ob es gelingt, den Gebrauch eines neuen Mediums in zunächst möglicherweise marginalen, dann zunehmend zentralen Bereichen der Gesellschaft zu verankern. Dies gilt und galt auch für die moderne Buchdruckgesellschaft, die gegen jede Autorität verstößt, die die Schriftgesellschaft im Umgang mit den Quellen und den Hierarchien mühsam zu einer eindrucksvoll geschlossenen Kosmologie aufgebaut hatte. Dass man sich auf dieses Teufelswerk der beweglichen Lettern und der Massenproduktion von Texten eingelassen hat, konnte zunächst nur dadurch gerechtfertigt werden, dass man vorgab, nur die Bibel massenhaft reproduzieren zu wollen und mit ihr die Erde so zu wässern, wie es sich Gott nicht besser wünschen könnte (Giesecke 1991).

Der Buchdruck galt als Maschine der Kommunikation - und das hieß zunächst Verbreitung der Bibel und weiterer gottesfürchtiger Literatur. Und niemand ahnte, dass das religiöse Angebot nicht ausreichen würde, genug Nachschub für die Druckmaschinen sicherzustellen, die mit einem erheblichen Kapitalaufwand in Betrieb genommen worden waren. Der Humanismus, die Aufklärung und der Gedanke einer Bildung für alle, inklusive der dafür erforderlichen Alphabetisierung, kam gerade recht, den fehlenden Content nachzuliefern und rezipierbar zu machen (Bäcker 2018).

Dirk Bäcker analysiert in eindrucksvoller Weise den Überschusssinn, den die digitalen Medien produzieren. Überschusssinn bedeutet dabei jeweils, dass ein Medium der Kommunikation mehr Möglichkeiten der Kommunikation bereitstellt, als je aktuell wahrgenommen werden können. Jede neue Medienepoche muss sich auf diesen Überschusssinn erst einstellen und das Einstellen heißt dabei nicht, dass der Überschusssinn verschwindet, sondern es heißt, das Formen bereitstehen, eben eine Struktur und eine Kultur der Gesellschaft, in denen er aufgegriffen und reduziert werden kann ohne ihn als solche zum Verschwinden zu bringen. Diese Formen sind Formen neuer gesellschaftlicher Kulturen, neuer gesellschaftlicher Zusammenhänge, die im Sinne einer Evolution entstehen, um mit Überschusssinn und Überschussinformationen umgehen zu können.

Dieses Entstehen ist ein im besten Sinne emergenter Prozess, in dem Selbstorganisation als Prinzip im Sinne Hakens wirksam wird. Durch die intensive Vernetzung durch das Internet werden Informationen im Überschuss bereitgestellt. Dadurch vernetzen sich Systeme miteinander und es entstehe neue Systeme. Zudem verändern bestehende Systeme ihre Phasenzustände und treten in Selbstorganisationsprozesse ein. Reden wir hier von Gesellschaft und gesellschaftlichen Herausforderungen, so muss mit Luhmann (1991) immer konstatiert werden, dass die Soziologie Gesellschaft systemtheoretisch als Kommunikationssystems versteht. Das heißt, wir reden von globalen Gesellschaften. Die Abhängigkeit der unterschiedlichen Ebenen, der unterschiedlichen gesellschaftlichen Teilsysteme, sowohl auf globaler als auf lokaler 
Ebene, die durch neue Medien miteinander vernetzt werden, werden auch in ihre Interdependenz durch Bronfenbrenners Ökosystemtheorie erklärt.

Bronfenbrenner (1981b) entwickelt mit seinem ökosystemtheoretischen Modell ein Modell, das Entwicklung als wechselseitigen, interaktionistischen Prozess zwischen dem Individuum und seiner sozialen Umwelt betrachtet. Dabei zieht ein Entwicklungsschritt den anderen nach sich, die Interaktionen sind ineinander verschachtelt und die verschiedenen Elemente des Systems beeinflussen sich wechselseitig. Die unterschiedlichen Systemebenen stehen also miteinander in Verbindung. Der ökosystemtheoretische Ansatz ist damit ein weiterer Erklärungsansatz, der zeigt, in welcher Weise Systeme miteinander in Beziehung und miteinander in Kommunikation stehen können. Diese Kommunikation und dieses in Beziehung stehen wird durch Digitalisierung verstärkt und ein Zusammenhang existiert zwischen den Systemen auf globaler Makroebene und lokaler individueller Mikroebene. Die Nutzung von Twitter zur Übermittlung politischer Kommunikation ist hierfür ein Beispiel, dass Ankündigungen oft direkt eine Kette an Einflüssen auslösen, die zunächst politisch, dann wirtschaftlich, dann direkt individuell fühlbar sind. durch den Der so entstehende Zusammenhang führt zu einer sich selbst beschleunigenden, selbsttätigen und nicht gerichteten, unvorhersehbaren, hochemergenten Weiterentwicklung in Teilbereichen der gesellschaftlichen Systeme.

\section{B 2.8 Autopoiesis und Selbstorganisation}

Die Autopoiesis nach Maturana (1987) versucht die nach dem zweiten Weltkrieg entstandene Kybernetik auf die Biologie zu übertragen. Maturanas Intention hierbei besteht darin, zu klären, wie der Mensch zur Erkenntnis gelangen kann. Nach Maturana sind lebende Systeme stets autopoietisch. Der Begriff Autopoiesis, (altgriechisch autos, deutsch selbst und poiein, deutsch schaffen, bauen) bedeutet so viel wie Selbsttun oder Selbstgestaltung. Demnach dürfen nur Systeme als autopoietisch bezeichnet werden, die ihre Systemelemente selbst erzeugen, also selbstorganisiert agieren. Alle Systemelemente müssen aus den vorhandenen Systemelementen entstehen. In diesem Zusammenhang spricht man von Zirkularität. Es werden keine Systemelemente aus der Umwelt in das System übernommen.

Autopoiesis ist auch ein Schlüsselbegriff in der soziologischen Systemtheorie von Niklas Luhmann, der den Begriff Autopoiesis auf die Betrachtung sozialer Systeme übertragen hat (Luhmann 1984). Er bezieht sich dabei auf das Werk von Maturana und Varela sowie die erweiternde Diskussion bei Milan Zeleny bezüglich der Anwendung des Konzeptes auf Organisationen (Zeleny 1981). Seine zentrale 
These lautet, dass soziale Systeme ausschließlich aus Kommunikation(ssystemen) bestehen und in Autopoiesis operieren. Darunter ist zu verstehen, dass die Systeme sich in einem ständigen, nicht zielgerichteten autokatalytischen Prozess quasi aus sich selbst heraus erschaffen. Die Systeme produzieren und reproduzieren demnach sich selbst (ebenda).

Autopoietische Systeme müssen abgeschlossen gegenüber der Umwelt sein. Damit ist gemeint, dass eine Strukturveränderung nur aus dem System heraus entstehen kann, Systeme also selbstreferentiell sind. Nicht gemeint ist damit eine energetische oder informationelle Abgeschlossenheit gegenüber der Umwelt. Denn Strukturänderungen auslösende Systemstörungen können durchaus durch Umwelteinflüsse erfolgen. Das System wählt durch die Festlegung der Systemgrenze den Umfang und die Art des Kontakts zur Umwelt. Diese Eigenschaft wird als strukturelle Kopplung bezeichnet und bedeutet, dass es eine Umweltkopplung zwischen dem System-Innen und dem System-Außen gibt, mit der der Systemumfang festgelegt ist. Aufgrund dieser Systemgrenze ist das System nicht fähig Zustandsänderungen der Umwelt wahrzunehmen. Auf der anderen Seite kann ein externer Beobachtender keine Aussagen über die interne Organisation des autopoietischen Systems treffen. Man bezeichnet dies als operative Geschlossenheit (ebenda). Von außen kann lediglich eine Betrachtung erfolgen.

Durch die operative Geschlossenheit und Selbstreferentialität autopoietischer Systeme ist eine gezielte Beeinflussung des Systems unmöglich. Da die Umwelt den Zustand des autopoietischen Systems nicht erkennen kann, kann die Umwelt nicht beurteilen, wie das System auf einen Umwelteinfluss, eine Störung reagiert. Der Einfluss in einem Organisationssystem oder einem Team von außen durch Informationsüberfluss, durch digitale Medien, kann also nach autopoietischer Analyse im System zu Änderungen führen, die aber selbstreferentiell und selbstorganisiert sind und insofern nicht im Ergebnis determiniert werden können.

Man spricht in der Autopoiesis von Selbstorganisation, da das autopoietische System spontan seinen eigenen Zustand an Randbedingungen der strukturellen Kopplung anpassen kann (ebenda). Die Autopoiesis hat im Bereich der Biologie und der Soziologie den Gedanken der Selbstorganisation etabliert. Eine Vielzahl von Managementpraktiken wurde von der Autopoiesis inspiriert. Der Bezug zur Emergenz ergibt sich, wenn man betrachtet, dass in einem autopoietischen System durch Selbsterzeugung und Selbstreferenz eine Vielzahl von Systemelementen organisiert werden und dabei in ihrer Gesamtheit (im emergenten Sinne) höhere oder neue Eigenschaften hervorbringen. In der Theorie der Autopoiesis wird betont, dass in einem autopoietischen System neben den Systemelementen eine systemspezifische Organisation herrscht. Dabei geht man davon aus, dass einzelne Systemelemente austauschbar sind, solange die spezifische Organisation erhalten 
bleibt. Darin zeigt sich, dass das Systemverhalten nicht auf das Verhalten der einzelnen Elemente zurückzuführen ist, sondern dass neben den Systemelementen eine spezifische Organisation entsteht, die für das Systemverhalten genauso entscheidend ist. Man kann deshalb davon ausgehen, dass autopoietische Systeme emergente Eigenschaften aufzeigen.

\section{B 2.9 Zusammenfassung und Fazit}

Im Fazit wird deutlich, dass das Zusammenwirken komplexer Systeme zur Selbstorganisation und Systemveränderung führt. Diese Systemveränderungen sind davon gekennzeichnet, dass sie nicht linear auf den vorherigen Zustand zurückzuführen sind und nicht deterministisch zustande kommen, also keine Vorhersagen getroffen werden können. Vernetzung durch digitale Medien, globales Zusammenwirken und der Überschuss von Informationen durch die Digitalisierung führen zu schnelleren Veränderungen auf der Ebene der sozialen Organisationen die sich auf allen Ebenen der Makro-, Meso- und Mikroebene noch einmal selbst verstärken und beschleunigen. Der Zusammenhang der Ökosystemebenen führt also zur Beschleunigung selbstorganisierter Veränderung.

Selbstorganisation liegt als Prinzip also vielen gesellschaftlichen Entwicklungen zugrunde. Es entwickelt sich zu einem so durchdringenden Konzept, dass wir die Entwicklung zur Selbstorganisation in der Gesellschaft insgesamt aber auch in den einzelnen gesellschaftlichen Teilbereichen, wie etwa den privaten oder öffentlichen Organisationen mit dem Begriff Drift to Self-Organisation bezeichnet haben.

Im nächsten Abschnitt wollen wir betrachten, in welcher Weise Selbstorganisation in Unternehmen und Organisationen wirkt. 